\title{
GMR
}

\section{A two-step method for identification of the Chinese glutinous rice Suyunuo, based on ISSR-SCAR and allele-specific markers}

\author{
Y.B. Lin ${ }^{1 *}$, Y.M. Zhang ${ }^{2,3 *}$, Y.Y. Hang, ${ }^{2,3}$, M.M. Li ${ }^{2,3}$, G.C. Zhou ${ }^{2,3}$, \\ X.L. Shen ${ }^{1}$ and X.Q. Sun ${ }^{2,3}$ \\ ${ }^{1}$ Seed Administrative Station of Suzhou, Suzhou, Jiangsu Province, China \\ ${ }^{2}$ Institute of Botany Jiangsu Province and Chinese Academy of Sciences, \\ Nanjing, China \\ ${ }^{3}$ The Jiangsu Provincial Platform for Conservation and Utilization of \\ Agricultural Germplasm, Nanjing, China \\ *These authors contributed equally to this study. \\ Corresponding authors: X.L. Shen / X.Q. Sun \\ E-mail: szseed@126.com / xiaoqinsun@cnbg.net
}

Genet. Mol. Res. 15 (4): gmr.15048663

Received March 28, 2016

Accepted July 11, 2016

Published October 5, 2016

DOI http://dx.doi.org/10.4238/gmr.15048663

Copyright (C) 2016 The Authors. This is an open-access article distributed under the terms of the Creative Commons Attribution ShareAlike (CC BY-SA) 4.0 License.

\begin{abstract}
Suyunuo is a valuable glutinous rice variety cultivated mainly in the Lake Taihu area of China. Historically, Suyunuo was presented to emperors as a tribute, and, still today, enjoys a great reputation in China. This study aimed to develop a unique, specific molecular marker for the identification of Suyunuo rice. Polymerase chain reaction (PCR) amplification of inter-simple sequence repeat (ISSR) molecular markers was performed on Suyunuo and 11 other glutinous rice varieties that are mainly cultivated in the Yangtze River Delta region. A Suyunuo-specific band was detected in the PCR products generated from primer ISSR-807. A sequence characterized
\end{abstract}


amplified region (SCAR) primer pair targeting a Suyunuo-specific band was subsequently designed. The SCAR primers amplified a target band in all individuals of Suyunuo and in four glutinous indica varieties, whereas no bands were found in the seven glutinous japonica varieties. Subsequently, sequences amplified by the SCAR primer pair were analyzed to facilitate the design of Suyunuo allele-specific primers. The allele-specific primer pair produced target bands in all individuals of Suyunuo rice but no bands in individuals of any of the other 11 rice varieties. This study provides a theoretical guideline for rice germplasm identification and innovation of other valuable rice landraces.

Key words: Suyunuo rice; Allele-specific primers; ISSR-SCAR; Cultivar identification; Two-step screening

\section{INTRODUCTION}

Rice (Oryza sativa L.) is an annual grain that is one of the world's staple crops, widely cultivated in subtropical areas. Many studies have been conducted to identify the diverse varieties of rice. In early studies, the identification and classification of rice varieties were mainly based on morphological characteristics, such as leaf shape, the number of leaf hairs, grain shape, grain length-to-width ratio, and physiological characteristics, such as serum and phenol reactions (Kato et al., 1928; Terao and Midusima, 1939; Matsuo, 1952; Cheng, 1985). Later studies used biochemical and physical methods, including isozyme analysis, high-performance liquid chromatography, gas chromatography-mass spectrometry, and nearinfrared spectroscopy, to distinguish among rice varieties (Glaszmann, 1987; Huebner et al., 1991; Wan and Ikehashi, 1997; Wu et al., 2012; Kong et al., 2013).

With the development of molecular biology techniques, DNA-based markers have been widely used in rice varietal identification, genetic-map construction, molecular breeding, and genetic diversity and relationship studies. For example, amplified fragment length polymorphisms, inter-simple sequence repeats (ISSRs), simple sequence repeats (SSRs), random amplified polymorphic DNAs, and other molecular markers have been used for fingerprinting varietal identification and to analyze genetic relationships among various Oryza species (Choudhury et al., 2001; Prashanth et al., 2002; Saini et al., 2004; Wu et al., 2009; Pourabed et al., 2015). In recent years, the development of sequence tagged sites, single nucleotide polymorphisms, and chloroplast and mitochondrial DNA sequences have allowed the evaluation and identification of rice seed quality and genetic diversity analyses (McNally et al., 2009; Lin et al., 2012; Okoshi et al., 2016). However, few studies have focused on determining the uniqueness or specificity of individual rice varieties.

Suyunuo, a valuable aromatic rice variety, has a long history of cultivation in the Lake Taihu area (Jiangsu Province of China). Historically, Suyunuo has often been presented to emperors as a tribute, and, still today, it enjoys a great reputation among Chinese consumers (Jiang et al., 1986). Most studies have considered Suyunuo a japonica variety (Jiang et al., 1986; Wang et al., 1990; He et al., 2006; Chen et al., 2008; Yan et al., 2011; Zhou et al., 2012; Huan et al., 2014), but some have categorized it as an indica variety (Lu, 1986; Shi, 1986; Yao and Wang, 1986). Based on its morphological characteristics, Suyunuo has many features consistent with japonica rice, such as a compact plant shape, glossy leaf surface,

Genetics and Molecular Research 15 (4): gmr.15048663 
low tiller to spike rate, and thick and dense glume pubescence (Jiang et al., 1986; Wang et al., 1990). However, other characteristics of Suyunuo, such as its tall plant height, light green leaf color, wide and long leaves, and especially its long grain shape, are consistent with indica rice (Lu, 1986; Shi, 1986; Yao and Wang, 1986). In addition to the classification based on morphological characteristics (Jiang et al., 1986; Lu, 1986; Shi, 1986; Yao and Wang, 1986; Wang et al., 1990), some studies have used molecular markers to help classify Suyunuo. For example, recent studies constructed DNA fingerprint maps of a collection of glutinous rice varieties including Suyunuo, based on SSR molecular markers (Zhu et al., 2012; Chen et al., 2014). However, specific markers for the efficient identification of Suyunuo are still lacking. Here, we screened a specific molecular marker and developed an efficient method for the authentication of Suyunuo.

\section{MATERIAL AND METHODS}

\section{Plant materials}

Twelve glutinous rice varieties widely cultivated in the Chinese Provinces of Jiangsu and Zhejiang, including Suyunuo, were chosen for the present study (Table 1). Suyunuo and Yaxuenuo are local varieties, whereas the others are breeding varieties.

\begin{tabular}{|c|c|c|}
\hline Code & Variety & Locality/Breeding unit \\
\hline 1 & Taihunuo & Suzhou Academy of Agricultural Sciences \\
\hline 2 & Zhenuo 5 & Zhejiang Academy of Agricultural Sciences \\
\hline 3 & Shaonuo 9714 & Shaoxing Academy of Agricultural Sciences \\
\hline 4 & Zhennuo 19 & Zhenjiang Academy of Agricultural Sciences \\
\hline 5 & Jinlingxiangnuo & Jiangsu Academy of Agricultural Sciences \\
\hline 6 & Zhendao 2 & Zhenjiang Academy of Agricultural Sciences \\
\hline 7 & Guanglingxiangnuo & Yangzhou University \\
\hline 8 & Yaxuenuo & Changshu local variety \\
\hline 9 & Guizhaonuo & Guangdong Academy of Agricultural Sciences \\
\hline 10 & Yangfunuo 4 & Yangzhou Academy of Agricultural Sciences \\
\hline 11 & Yangxiannuo 32-2 & Yangzhou Academy of Agricultural Sciences \\
\hline 12 & Suyunuo & Suzhou local variety \\
\hline
\end{tabular}

Ten individuals were used for each variety.

\section{Extraction of total genomic DNA}

Genomic DNA was extracted from 10 individual seedlings randomly selected from each variety. After sowing, once seedlings grew to a height of 10 to $15 \mathrm{~cm}, 100 \mathrm{mg}$ fresh leaves was collected for DNA extraction. Leaf samples were disrupted with a Tissue Lyser LT (Qiagen, Hilden, Germany) and DNA was extracted using an Easy Pure Plant Genomic DNA Kit (TransGen Biotechnology Co., Ltd., Beijing, China) following the manufacturer instructions. The concentration and purity of DNA samples were determined using a nucleic acid and protein detector (Eppendorf BioPhotometer D30, Hamburg, Germany). The DNA was adjusted to a concentration of $20 \mathrm{ng} / \mu \mathrm{L}$ and was stored at $-20^{\circ} \mathrm{C}$ until further analysis.

\section{Selection of ISSR primers}

The ISSR primers used in the present study have been reported in previous studies

Genetics and Molecular Research 15 (4): gmr.15048663 
(Saini et al., 2004; Jeung et al., 2005) and were synthesized by Nanjing Ruizhen Biological Technology Co., Ltd. (Nanjing, China). Equal amounts of DNA from 10 individuals of each of the 12 glutinous rice varieties were mixed to generate DNA pools for each variety, which were then used as a polymerase chain reaction (PCR) template. The PCRs were performed on a thermal cycler (Biometra T1 Thermocycler, Göttingen, Germany). The optimized ISSR amplification conditions consisted of a total reaction volume of $20 \mu \mathrm{L}$, including $1.0 \mu \mathrm{L}$ DNA template $(20 \mathrm{ng} / \mu \mathrm{L}), 10.0 \mu \mathrm{L} 2 \mathrm{X}$ reaction mix (containing $20 \mathrm{mM}$ Tris- $\mathrm{HCl}, 100 \mathrm{mM} \mathrm{KCl}, 3$

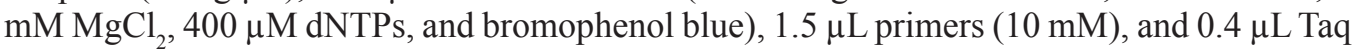
DNA polymerase $(2.5 \mathrm{U} / \mu \mathrm{L})$; double-distilled water was added to produce a final volume of $20 \mu \mathrm{L}$. The PCR amplification program was as follows: $94^{\circ} \mathrm{C}$ for $5 \mathrm{~min} ; 40$ cycles of $94^{\circ} \mathrm{C}$ for $1 \mathrm{~min}, 50^{\circ}-53^{\circ} \mathrm{C}$ (different primers had different annealing temperatures) for $1 \mathrm{~min}$, and $72^{\circ} \mathrm{C}$ for $1.5 \mathrm{~min}$; followed by $72^{\circ} \mathrm{C}$ for $10 \mathrm{~min}$. The PCR products were analyzed by electrophoresis on a $2 \%$ agarose gel that was run at $80 \mathrm{~V}$ for $1.5 \mathrm{~h}$, followed by $0.1 \%$ ethidium bromide (EB) staining and visualization under UV light. Band size was estimated from a DL2000 DNA ladder (Guangzhou Dongsheng Biotechnology Co., Ltd., Guangzhou, China).

\section{Cloning and sequencing of ISSR-sequence characterized amplified region (SCAR)- specific bands}

The specific bands detected in the Suyunuo sample were extracted from the gel, and subcloned into a pMD19-T vector (TaKaRa Biotechnology Co., Ltd., Dalian, China) by TA cloning methods. The recombinant vectors were transformed into Escherichia coli DH5a competent cells (TaKaRa Biotechnology Co., Ltd.) following the manufacturer instructions. Single colonies were picked for a colony PCR test, and positive clones with the specified band were sequenced by Nanjing Ruizhen Biological Technology Co., Ltd. Based on the results of the DNA sequence analysis, a pair of specific primers was designed for the subsequent SCAR reaction using the Primer Premier v. 5.0 software.

\section{Verification of ISSR-SCAR markers}

The synthesized SCAR primers were used to verify 10 samples of each of the 12 rice varieties. The reaction volume was $20 \mu \mathrm{L}$, and the components and final concentrations included $1.0 \mu \mathrm{L}$ DNA template $(20 \mathrm{ng} / \mu \mathrm{L}$ ), $10.0 \mu \mathrm{L} 2 \mathrm{X}$ reaction mix (containing $20 \mathrm{mM}$ Tris- $\mathrm{HCl}, 100 \mathrm{mM} \mathrm{KCl}, 3 \mathrm{mM} \mathrm{MgCl}, 400 \mu \mathrm{M}$ dNTPs, and bromophenol blue), $1.0 \mu \mathrm{L}$ each primer $(10 \mathrm{mM}), 0.4 \mu \mathrm{L}$ Taq DNA polymerase $(2.5 \mathrm{U} / \mu \mathrm{L})$; double-distilled water was added to produce a final volume of $20 \mu \mathrm{L}$. The amplification program was performed as follows: $94^{\circ} \mathrm{C}$ for $5 \mathrm{~min} ; 30$ cycles at $94^{\circ} \mathrm{C}$ for $45 \mathrm{~s}, 55^{\circ} \mathrm{C}$ for $45 \mathrm{~s}$, and $72^{\circ} \mathrm{C}$ for $1 \mathrm{~min}$; and, finally, $72^{\circ} \mathrm{C}$ for $10 \mathrm{~min}$. The PCR products were analyzed by electrophoresis on a $1 \%$ agarose gel that was run at $80 \mathrm{~V}$ for $30 \mathrm{~min}$ and stained with EB. The products were visualized and photographed by a gel imaging system, as described above.

\section{Design and verification of Suyunuo-specific molecular markers}

Suyunuo and the other four rice varieties that resulted in an amplified target band generated by using the ISSR-SCAR primers were amplified by PCR and sequenced using the developed primer pair. The sequencing results were aligned, and a Suyunuo-specific primer

Genetics and Molecular Research 15 (4): gmr.15048663 
pair was designed to target the sites that differentiated Suyunuo from the other rice varieties. This primer pair was then used to verify specificity by evaluating 10 individual seedling samples from each of the 12 varieties. The PCR program and conditions were the same as that for ISSR-SCAR marker verification with the exception of an annealing temperature of $60^{\circ} \mathrm{C}$.

\section{RESULTS}

When a collection of ISSR primers was used to perform PCR amplification on the DNA pools from 12 glutinous rice varieties, one primer, ISSR-807 (5'-AGAGAGAGAGAGAGAGT-3'), was noted for its ability to specifically identify Suyunuo. One of the amplification products of ISSR-807, a band of about $1200 \mathrm{bp}$, was specific to Suyunuo (highlighted with an arrow in Figure 1). Cloning and sequencing of the Suyunuo-specific fragment amplified by ISSR-807 showed that the total fragment length was 1154 bp. The resulting nucleotide sequence is shown in Figure 2. An SCAR primer pair was designed (underlined in Figure 2) (F1: 5'-AGAGAGTGAAGGGGAAGAGATA-3' and R1: 5'-CCTCCGTATTAAAGTAAGTTCG-3'), which corresponded to the 11-32 and 897-918 nt regions of the Suyunuo-specific fragment sequence, respectively.

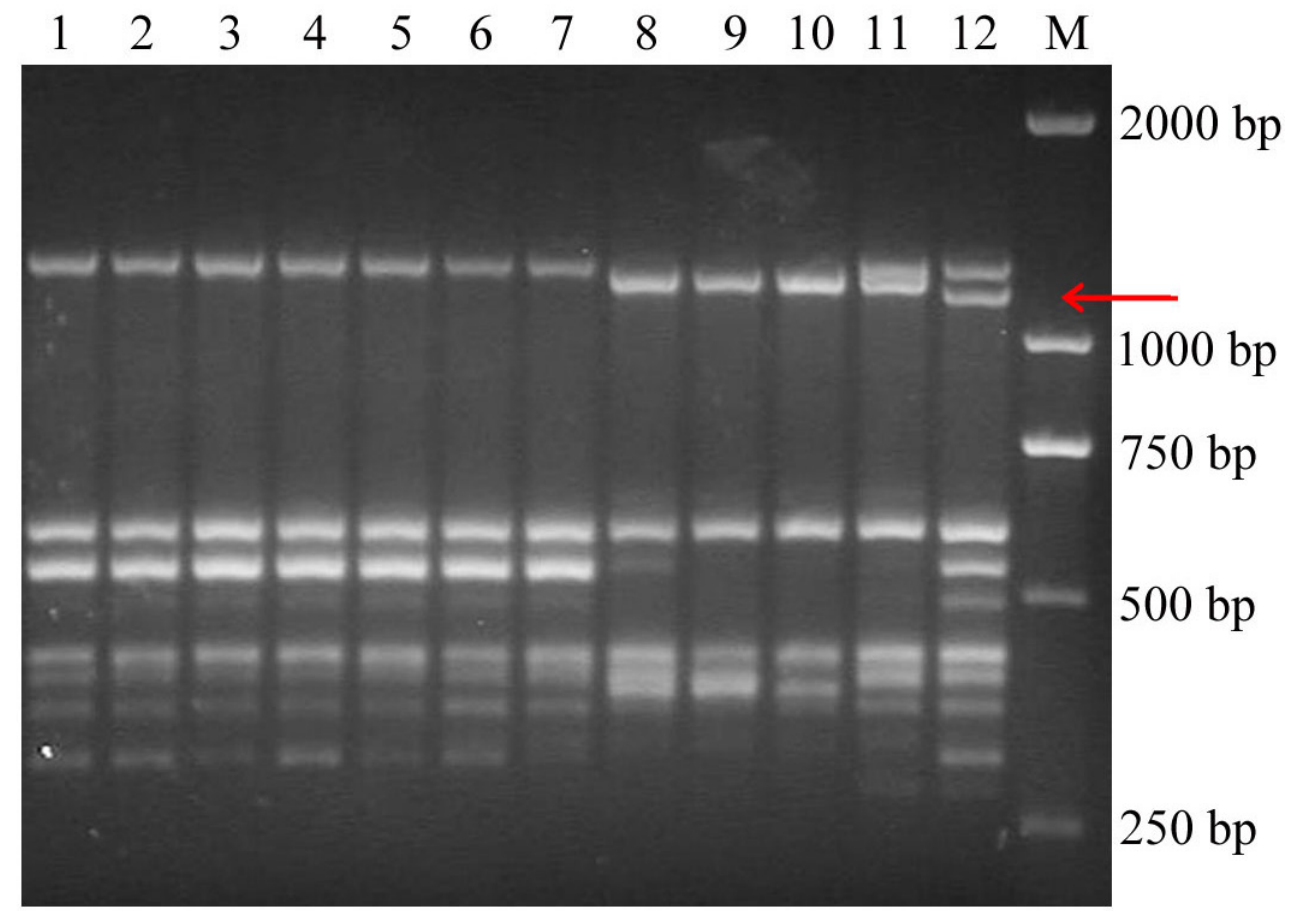

Figure 1. Agarose gel electrophoresis of PCR products obtained when using the primer ISSR-807 among 12 Oryza sativa L. varieties. Lanes 1-12 are Taihunuo, Zhenuo 5, Shaonuo 9714, Zhennuo 19, Jinlingxiangnuo, Zhendao 2, Guanglingxiangnuo, Yaxuenuo, Guizhaonuo, Yangfunuo 4, Yangxiannuo 32-2, and Suyunuo varieties, respectively. Lane M, DL2000 DNA ladder; the red arrow represents the Suyunuo-specific band. 


\begin{abstract}
5'-AGAGAGAGAGAGAGAGTGAAGGGGAAGAGATAAAGGAGAGGATAAGACTGAATA TGTGGTTGGCGAGCGAGAGGATATTTATAGTAGTAGTTATCCCGTACACTGTCCCATA GTTTTTCTGAAAGGCATTTGATTACTTATTGGTTGCATGTTCATTAGTTCTTCTTTAGG AAACTACGGCGGGTTTGAACACCAGCCTGAACTTTATTCATATGGTAAATAAACTTTA CAAAGGGGGGAGGTACAGAGGGACGGGGAGAAGAAACTATCATTATTTTTATCACAT TGTGAATGAATTAATTACTTATTAGTCTTGGTGCCAAAAGAAATAGGTCTTAGATGCG AAAGGGCCTTTAGCCTAATGGTTACAAGAACCTCAGTAGTACCTGAGGTCCTGGGTTC GACTCCCCATAGGAGCGAATTTTCCAGGATTTAATGGTGTTTGTGCTTTCAGTGGTAG GCGACGCACCCGTCGACAGCGAGGCGCCTGTGGTGACTTCGTCAATCTCTCCAGAATT TGCCGACCCAGTCTTCAAAGATGCTCATAGGGGTAGGGTTTGCGTGCGTGTGTTCAGA GGGTACGTCGCCAGCGAGGCGCCTGTGGGGGGTAGGGTTTGCATGCTTGTGTTCATA GGGTACGTCGCCAGCGAGGCGCCTGTGGTGACTTCGTCAACCTCTCCAGAATTTGCCA GCCCAGTCTTCGAAGTGAGTAGCGTTGTACTGTGTAATTTAAAAAAAATAGGTCTTAG ATTTTTGGATGAAAGGAGTATAAAAGTAGAGAATAAATATATTGAAGTATAAAATGG AAAAAAAACTATATTTAGATTTGATAAAATGGAAATATTCTATTATCTTTTTATGCTG CATTAATATGTGTCATGTGTGATATTGAATAACGAACTTACTTTAATACGGAGGAGTA TTTTCCTTGAACCGGGATGATGAAATCATTGAAAACGCTGAGGCAAGAGGACGTGCA ACACGTGGGATAGCGCCGACGTCGGCGATCTGTTCTACCTTCTGGAAAACAAGTCTG CAAAAATTAATTTCTGCCTGAGGCAGTGAGGCTCGCTTGTCAGTCACCCGTATACGTG GAAAATAAATTTTCTCTCGCTTTCTCACGCGGGTCGCGAGGAACTCTCTCTCTCTCTCT A-3'
\end{abstract}

Figure 2. Nucleotide sequence of the Suyunuo-specific fragment amplified when using primer ISSR-807. The underlined sequences were used to design SCAR primers.

The designed SCAR primer pair was used to analyze DNA samples from 120 individual seedlings, representing the 12 glutinous rice varieties. Our results showed that one distinct band of about $900 \mathrm{bp}$ was amplified in all individuals of five rice varieties: Suyunuo, Yaxuenuo, Guizhaonuo, Yangfunuo 4, and Yangxiannuo 32-2. This specific band was not amplified in any individuals from the other seven rice varieties (Figure 3). These results suggest that the ISSR-SCAR marker can only distinguish Suyunuo from a subset of the other glutinous varieties and, thus, cannot be used specifically for the identification of Suyunuo.

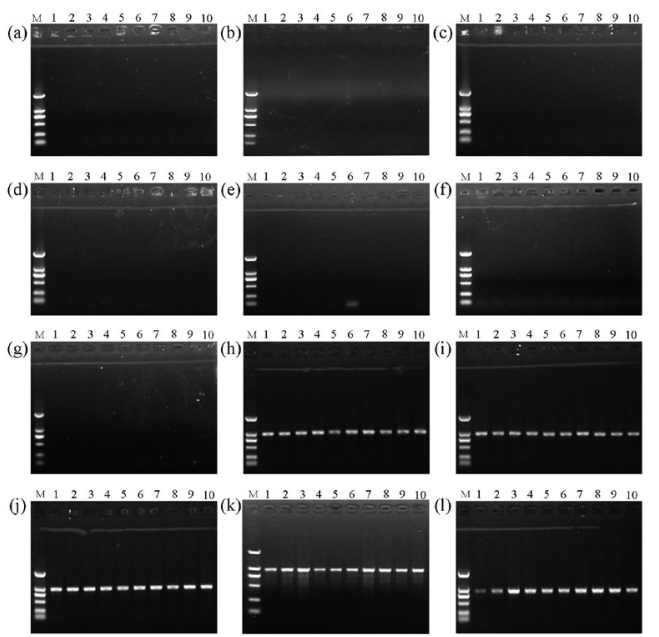

Figure 3. Verification of the ISSR-SCAR by PCR of 10 individuals from each of the 12 Oryza sativa L. varieties. Panels a-1 show the results of PCR in ten (lanes 1-10 in each figure) plants of each variety from Taihunuo, Zhenuo 5, Shaonuo 9714, Zhennuo 19, Jinlingxiangnuo, Zhendao 2, Guanglingxiangnuo, Yaxuenuo, Guizhaonuo, Yangfunuo 4, Yangxiannuo 32-2, and Suyunuo, respectively. Lane M, DL2000 DNA ladder. 
The specific PCR fragments amplified in Suyunuo, Yaxuenuo, Guizhaonuo, Yangfunuo 4, and Yangxiannuo 32-2 were sequenced. Our sequence alignment (Figure 4) showed that the nucleotide sequence of Suyunuo contained a unique 42 nt deletion at 214-255 bp. Further BLAST in the NCBI database (http://blast.ncbi.nlm.nih.gov/Blast.cgi) revealed that the $42 \mathrm{nt}$ deletion was not detected in the whole genome sequences of japonica or indica rice. Therefore, the sequence encompassing this deletion provided a unique target site for designing a Suyunuospecific primer. Consequently, a pair of allele-specific primers was designed for specific amplification of Suyunuo. Their sequences were: F2 (5'-AGTACAACGCTACTCACTTC-3') and R2 (5'-GGACGGGGAGAAGAAACTATCA-3'), with the forward primer (F2) located at the boundary of the deletion (as indicated in Figure 4). We then again evaluated the 120 individual plants representing all 12 glutinous rice varieties with the second designed Suyunuo allele-specific primer. The results showed that one distinct band of about $500 \mathrm{bp}$ was amplified in all 10 Suyunuo individuals but was not observed in any individuals of the other 11 varieties (Figure 5).

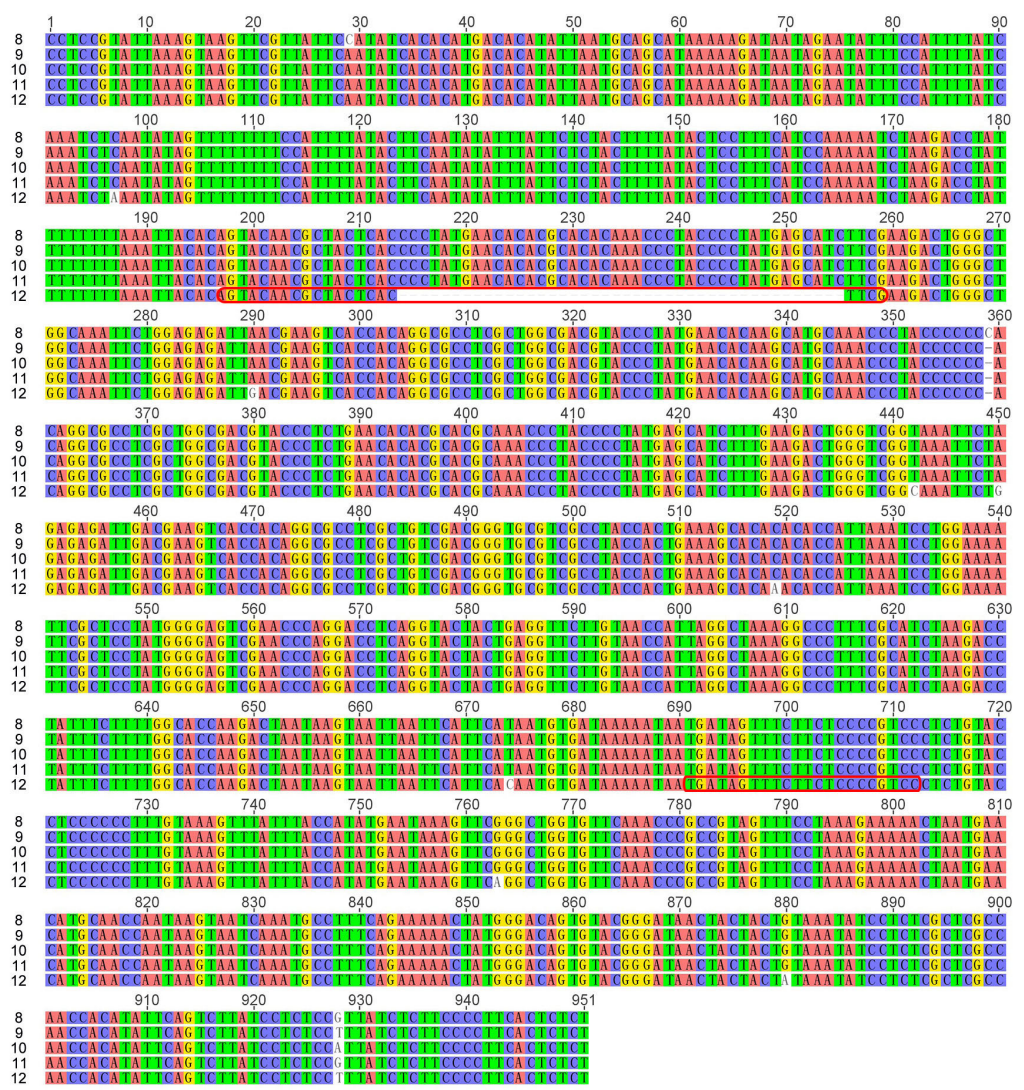

Figure 4. Alignment of amplified sequences from Yaxuenuo, Guizhaonuo, Yangfunuo 4, Yangxiannuo 32-2, and Suyunuo generated by using ISSR-SCAR primers. The designed allele-specific primer sequences are identified by red boxes.

Genetics and Molecular Research 15 (4): gmr.15048663 

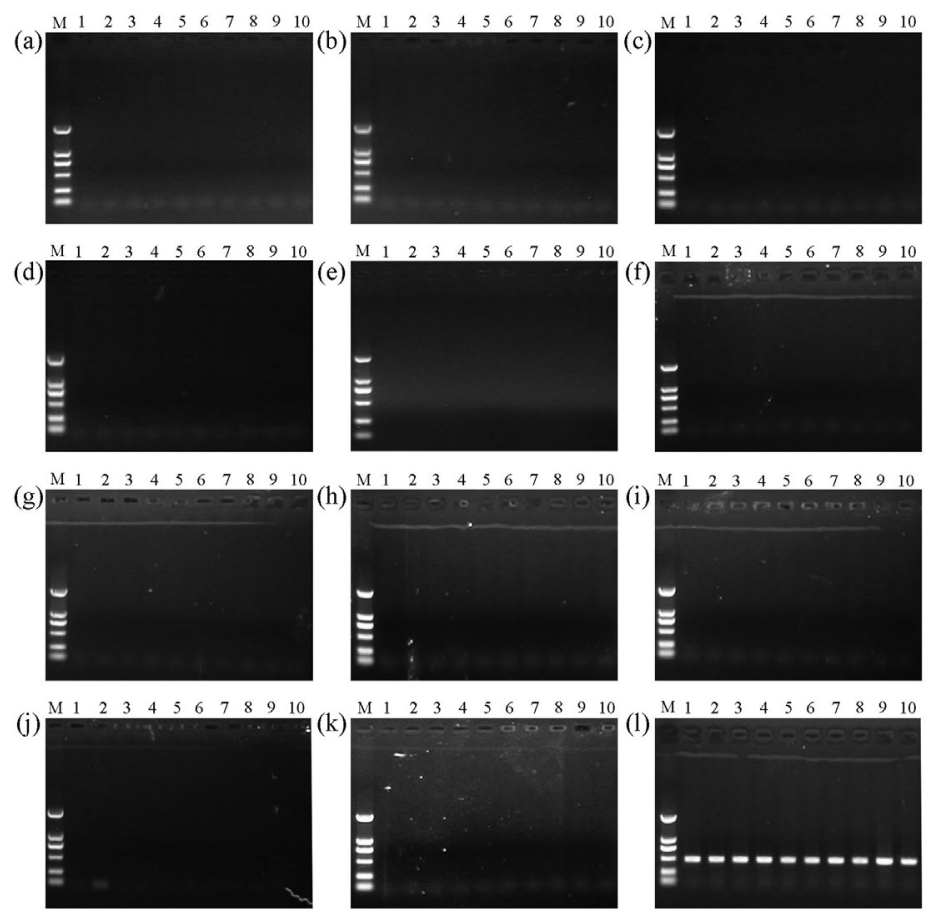

Figure 5. PCR verification of the Suyunuo-specific allele-specific primers. Panels a-1 show the results of PCR in the ten (lanes 1-10 in each figure) plants of each variety from Taihunuo, Zhenuo 5, Shaonuo 9714, Zhennuo 19, Jinlingxiangnuo, Zhendao 2, Guanglingxiangnuo, Yaxuenuo, Guizhaonuo, Yangfunuo 4, Yangxiannuo 32-2, and Suyunuo, respectively. Lane $M$, DL2000 DNA ladder.

\section{DISCUSSION}

According to the previous studies (He et al., 2010; Yan et al., 2011) and the documentation in the National Rice Data Center (http://www.ricedata.cn/variety), among the glutinous rice varieties that we evaluated, Taihunuo, Zhenuo 5, Shaonuo 9714, Zhennuo 19, Jinlingxiangnuo, Zhendao 2, Guanglingxiangnuo, and Suyunuo can be classified as japonica varieties, whereas Yaxuenuo, Guizhaonuo, Yangfunuo 4, and Yangxiannuo 32-2 can be classified as indica varieties.

The SCAR marker, designed from our ISSR-807 amplification results, amplified the target band in all four indica varieties and Suyunuo, but did not produce a band in the seven japonica varieties, suggesting that this SCAR marker can correctly classify Chinese glutinous rice varieties to subspecies (japonica and indica). Suyunuo, which was thought to be a japonica type, showed PCR results consistent with indica varieties. One explanation for this is that, although Suyunuo has been considered to be a japonica variety in the majority of past studies (Jiang et al., 1986; Wang et al., 1990; He et al., 2006; Chen et al., 2008; Yan et al., 2011; Zhou et al., 2012; Huan et al., 2014), it possesses many typical characteristics of indica rice, including plant shape, leaf shape and color, and grain shape. Consistent with our findings, a previous study by Zhu et al. (2012) investigating the genetic relationships based on SSRs also showed that Suyunuo is closely related to the indica variety Yangdao 6. 
The allele-specific PCR primers designed based on the PCR fragment sequence results of Suyunuo and the four indica varieties only amplified the target band in Suyunuo but no other variety, indicating that the designed allele-specific PCR primer pair is a promising candidate for distinguishing Suyunuo from other rice varieties. We further blasted the amplified Suyunuo sequence (Figure 4) against whole genome sequences of japonica and indica rice and found that the $42 \mathrm{nt}$ deletion between 214 and $255 \mathrm{bp}$ in Suyunuo was not detected in the genome sequences of either japonica or indica rice, indicating that this deletion may be characteristic of Suyunuo. This could be a natural mutation that occurred during the long-term cultivation and domestication of this variety.

In the present study, we developed an allele-specific PCR molecular marker for Suyunuo identification based on ISSR-SCAR markers. This marker could successfully distinguish Suyunuo from 11 other glutinous rice varieties planted in the Jiangsu and Zhejiang Provinces, and the identification efficiency was 100\% for the 10 detected Suyunuo individuals. Compared to the traditional morphological, physical, chemical indicators, and non-specific molecular markers, this PCR-based identification technology presents the advantages of high accuracy, good reproducibility, stability, and reliability. Since only glutinous rice varieties that are popularly cultivated in the Yangtze River Delta region were included in this study, further research is needed to confirm the efficiency of the molecular marker when more rice varieties are included. However, the development of a candidate specific molecular marker for Suyunuo in this study provides a theoretical basis and guideline for rice germplasm identification and innovation of other valuable rice landraces.

\section{Conflicts of interest}

The authors declare no conflict of interest.

\section{ACKNOWLEDGMENTS}

Research supported by the Foundation Project of Suzhou Municipal Science and Technology Bureau (\#SZP201313) and the Ability Improvement Project of Jiangsu Social Scientific Research Institutions (\#BM2015019).

\section{REFERENCES}

Chen P, Huang J, Zhu Y, Qiao Z, et al. (2014). Establishment of DNA fingerprinting with SSR for 'Taihuno', 'Suyuno' and some other glutinous rice varieties. Zhongguo Nongxue Tongbao 30: 137-141.

Chen S, Yang Y, Shi W, Ji Q, et al. (2008). Badh2, encoding betaine aldehyde dehydrogenase, inhibits the biosynthesis of 2-acetyl-1-pyrroline, a major component in rice fragrance. Plant Cell 20: 1850-1861. http://dx.doi.org/10.1105/ tpc. 108.058917

Cheng KS (1985). A statistical evaluation of the classification of rice cultivars into hsien and keng subspecies. Rice Genet. Newsl. 2: 46-48.

Choudhury PR, Kohli S, Srinivasan K, Mohapatra T, et al. (2001). Identification and classification of aromatic rices based on DNA fingerprinting. Euphytica 118: 243-251. http://dx.doi.org/10.1023/A:1017554600145

Glaszmann JC (1987). Isozymes and classification of Asian rice varieties. Theor. Appl. Genet. 74: 21-30. http://dx.doi. org/10.1007/BF00290078

He XF, Shu CL, Liu LL, Wang F, et al. (2010). Difference of amylopectin structure among various rice genotypes differing in grain qualities and its relation to starch physicochemical properties. Acta Agron. Sin. 36: 276-284. http://dx.doi. org/10.1016/S1875-2780(09)60035-3

Genetics and Molecular Research 15 (4): gmr.15048663 
He Y, Han Y, Jiang L, Xu C, et al. (2006). Functional analysis of starch-synthesis genes in determining rice eating and cooking qualities. Mol. Breed. 18: 277-290. http://dx.doi.org/10.1007/s11032-006-5505-7

Huan J, Bao YM, Wu YY, Zeng GY, et al. (2014). Identification of quantitative trait loci conferring blast resistance in Bodao, a japonica rice landrace. Genet. Mol. Res. 13: 9756-9765. http://dx.doi.org/10.4238/2014.November.27.3

Huebner FR, Hussain A, Lookhart GL, Bietz JA, et al. (1991). Discrimination of sister line IR rice varieties by polyacrylamide gel electrophoresis and reversed phase high-performance liquid chromatography. Cereal Chem. 68: 583-588.

Jeung JU, Hwang HG, Moon HP and Jena KK (2005). Fingerprinting temperate japonica and tropical indica rice genotypes by comparative analysis of DNA markers. Euphytica 146: 239-251. http://dx.doi.org/10.1007/s10681-005-9022-2

Jiang H, Wu J and Wang G (1986). Famous and excellent rice resource - Suyunuo. China Seed Ind. 1: 37.

Kato S, Kosaka H and Hara S (1928). On the affinity of rice varieties as shown by the fertility of hybrid plants. Bull. Sci. Fac. Agric. Kyushu Univ. 3: 132-147.

Kong W, Zhang C, Liu F, Nie P, et al. (2013). Rice seed cultivar identification using near-infrared hyperspectral imaging and multivariate data analysis. Sensors 13: 8916-8927. http://dx.doi.org/10.3390/s130708916

Lin HY, Wu YP, Hour AL, Ho SW, et al. (2012). Genetic diversity of rice germplasm used in Taiwan breeding programs. Bot. Stud. 53: 363-376.

Lu K (1986). Suyunuo. Crops 1: 38.

Matsuo T (1952). Gene ecological studies on the cultivated rice. Bull. Natl. Inst. Agr. Sci. 3: 1-111.

McNally KL, Childs KL, Bohnert R, Davidson RM, et al. (2009). Genomewide SNP variation reveals relationships among landraces and modern varieties of rice. Proc. Natl. Acad. Sci. USA 106: 12273-12278. http://dx.doi.org/10.1073/ pnas.0900992106

Okoshi M, Matsuno K, Okuno K, Ogawa M, et al. (2016). Genetic diversity in Japanese aromatic rice (Oryza sativa L.) as revealed by nuclear and organelle DNA markers. Genet. Resour. Crop Evol. 63: 199-208. http://dx.doi.org/10.1007/ $\underline{\text { s10722-015-0239-1 }}$

Pourabed E, Jazayeri Noushabadi MR, Jamali SH, Moheb Alipour N, et al. (2015). Identification and DUS testing of rice varieties through microsatellite markers. Int. J. Plant Genomics 2015: 965073. http://dx.doi.org/10.1155/2015/965073

Prashanth SR, Parani M, Mohanty BP, Talame V, et al. (2002). Genetic diversity in cultivars and landraces of Oryza sativa subsp. indica as revealed by AFLP markers. Genome 45: 451-459. http://dx.doi.org/10.1139/g02-003

Saini N, Jain N, Jain S and Jain RK (2004). Assessment of genetic diversity within and among Basmati and non-Basmati rice varieties using AFLP, ISSR and SSR markers. Euphytica 140: 133-146. http://dx.doi.org/10.1007/s10681-004$\underline{2510-\mathrm{y}}$

Shi J (1986). Glutinous rice cultivar Suyunuo. Seed World 9: 10 .

Terao H and Midusima U (1939). Some consideration on the classification of Oryza sativa L. into two subspecies so-called 'Japonica' and 'Indica'. Jap. J. Bot. 10: 213-258.

Wan J and Ikehashi H (1997). Identification of two types of differentiation in cultivated rice (Oryza sativa L.) detected by polymorphism of isozymes and hybrid sterility. Euphytica 94: 151-161. http://dx.doi.org/10.1023/A:1002907421839

Wang G, Jiang H, Wu J and Jiang G (1990). Special rices - Suyunuo and Longjing 4. Biol. Spec. 4: 14-15.

Wu J, Yu H, Dai H, Mei W, et al. (2012). Metabolite profiles of rice cultivars containing bacterial blight-resistant genes are distinctive from susceptible rice. Acta Biochim. Biophys. Sin. 44: 650-659. http://dx.doi.org/10.1093/abbs/gms043

Wu Y, Zhang Z, Chen Y, Wang B, et al. (2009). Authentication of Thailand jasmine rice using RAPD and SCAR methods. Eur. Food Res. Technol. 229: 515-521. http://dx.doi.org/10.1007/s00217-009-1072-6

Yan CJ, Tian ZX, Fang YW, Yang YC, et al. (2011). Genetic analysis of starch paste viscosity parameters in glutinous rice (Oryza sativa L.). Theor. Appl. Genet. 122: 63-76. http://dx.doi.org/10.1007/s00122-010-1423-5

Yao Y and Wang J (1986). Cultivation techniques of special rice Suyunuo in Taihu lake area. Jiangsu Agric. Sci. 3: 11-12.

Zhou Y, Yuan Y, Yuan F, Wang M, et al. (2012). RNAi-directed down-regulation of RSV results in increased resistance in rice (Oryza sativa L.). Biotechnol. Lett. 34: 965-972. http://dx.doi.org/10.1007/s10529-012-0848-0

Zhu Y, Huang L, Xie Y, Wu J, et al. (2012). Construction of SSR DNA fingerprints of Suxiangjing 1. Jiangsu Agric. Sci. 40: 34-35.

Genetics and Molecular Research 15 (4): gmr.15048663 